\title{
Monomorium moathi sp. n., a new ant species from Yemen (Hymenoptera: Formicidae) related to the salomonis-group
}

\author{
Abdulrahman S. Aldawood ${ }^{1}$; Mostafa R. Sharaf ${ }^{1}$; Cedric A. Collingwood $^{2}$ \\ 1- Plant Protection Department, College of Food and Agriculture Sciences, King Saud University, \\ Riyadh 11451, PO Box 2460, Kingdom of Saudi Arabia. \\ 2- 18 Milton street, Skipton, North Yorkshire, BD 23 2ED, U.K.
}

\begin{abstract}
Monomorium moathi sp. $\mathrm{n}$. is described and illustrated from Yemen based on the worker caste. This species belongs to the Monomorium salomonis-group, with closest resemblance to M. areniphilum Santschi, 1911. It is distinguished from the latter species by the following characters: Eyes oval, relatively large with eleven ommatidia in the longest row; Petiole node high and pointed in profile; Head, mesosoma and waist distinctly shagreenate granulate. Gaster finely shagreenate. Head dorsum, mesosoma, petiole, postpetiole and gastral tergites without hairs.
\end{abstract}

Keywords: Arabia, Palaearctic, Saudi Arabia, Myrmicinae, salomonis group, Taxonomy.

\section{INTRODUCTION}

Monomorium was established by Mayr in 1855 by the type species Monomorium minutum Mayr, 1855 (later, given the new name Monomorium monomorium Bolton (1987:287). This genus includes 296 species and subspecies (Bolton, 1995) distributed in all zoogeographical regions, but the majority of species occur in the Old World tropics and warm temperate regions (Brown, 2000). Most species nest in rotten woods, under rocks or directly on earth.

Within the subfamily Myrmicinae, workers of the genus Monomorium are easily recognized by the combination of the following characters: Monomorphic to polymorphic ants, mandibles with 3-5 teeth which decrease in size from apex to base. Median portion of clypeus raised, the raised section longitudinally bicarinate. Frontal carinae absent behind frontal lobe. Antennal scrobes absent. Antennae 10-12 segmented but most frequently 12, usually with a conspicuous 3-segmented club. Eyes present but sometimes reduced to a single ommatidium as in the fossulatumgroup. Metanotal groove present. Propodeal dorsum usually unarmed and rounding into the declivity. Petiole pedunculate anteriorly with a small anteroventral process.

The M. salomonis group (Bolton, 1987) is distinguished from other Monomorium species-groups by the following characters: Monomorphic with some size variation in any series. Palp formula 2,2. mandibles sculptured. Masticatory margin of mandibles with 4 teeth. Eyes distinct and moderate to large in size, circular to roughly oval, with 6 or more ommatidia in the longest row. Head always longer than broad. Scapes usually relatively large. Metanotal groove moderately impressed to absent. Propodeal dorsum usually sculptured but never transversely striate. Alitrunk, petiole and postpetiole usually conspicuously sculptured.

\footnotetext{
${ }^{1}$ Author to whom correspondence should be addressed. E-mail: antsharaf@yahoo.com
} 
The ant fauna of the genus Monomorium in Yemen consists of 23 species scattered in Collingwood (1985), Collingwood \& Van Harten (1994), Collingwood \& Agosti (1996), Collingwood \& Van Harten (2001), and Collingwood et al. (2004); 11 of them being described as new. In spite of these studies, species of that genus in Yemen are still inadequately known. In fact, this low number of Monomorium species in Yemen does not reflect the real number actually present as Yemen is considered one of the most poorly collected countries and little is known about its ant fauna, which is nearly afrotropical. In the present study we describe a new species of the genus Monomorium, M. moathi sp. n. from Yemen.

\section{MEASUREMENTS AND INDICES}

Measurements are in $\mathrm{mm}$ and indices are as follows:

$\mathrm{TL}=$ Total Length; the outstretched length of the ant from the mandibular apex to the gastral apex.

HW = Head Width; the maximum width of the head behind eyes in full face view.

$\mathrm{HL}=$ Head Length; the maximum length of the head, excluding the mandibles.

$\mathrm{CI}=$ Cephalic Index (HW x 100/HL).

$\mathrm{SL}=$ Scape Length, excluding basal neck.

SI $=$ Scape Index (SL x 100/HW).

$\mathrm{EL}=$ Eye Length; the maximum diameter of the eye.

$\mathrm{ML}=$ Mesosoma Length; the length of the mesosoma in lateral view, from the point at which the pronotum meets the cervical shield to the posterior base of the propodeal lobes or teeth.

PRW $=$ The maximum width of the pronotum in dorsal view.

$\mathrm{PL}=$ Petiole Length; the maximum length measured in dorsal view, from the anterior margin to the posterior margin.

$\mathrm{PW}=$ Petiole Width; maximum width measured in dorsal view.

PPL $=$ Postpetiole Length; maximum length measured in dorsal view.

$\mathrm{PPW}=$ Postpetiole Width; maximum width measured in dorsal view .

All measurements were given in millimeters and follow the standard measurements (Bolton, 1987).

Monomorium moathi Sharaf \& Collingwood sp. n. (Figs.1 \& 2)

Holotype. worker, Yemen, unknown locality, 1999 (C. A. Collingwood)), King Saud Museum of Arthropods (KSMA).

Measurements: Holotype worker: TL: 2.41; HL: 0.65; HW: 0.52; SL: 0.52; ML: 0.75; PRW: 0.35; EL: 0.12; PL: 0.18; PW: 0.19; PPL: 0.11; PPW: 0.19 Indices: CI: 80; SI: 100.

Paratypes: 3 workers; TL: 2.48; HL: 0.61-0.65; HW: 0.48-0.52; SL: 0.460.52; ML:0.63-0.78; PRW: 0.28-0.35; EL: 0.11-0.12; PL: 0.19-0.22; PW: 0.17-0.26; PPL: 0.12-0.14; PPW: 0.19 Indices: CI: 80-83; SI: 96-100 (one paratype is sound and the other two with broken gasters, same data as holotype).

Diagnosis. This new species is distinguished by the combination of the following characters: Colour brown, gaster blackish with characteristic bluish or violet reflection, general appearance dull. Eyes oval with eleven ommatidia in the longest row. Metanotal groove shallow but distinct. Head, mesosoma and waist distinctly shagreenate granulate. Gaster finely shagreenate. Body lacking pilosity except few short hairs in front of eyes, one or two long hairs on head ventrum and many long hairs on gastral ventrum. 
Description of worker. Body brown, gaster blackish with characteristic bluish or violet reflection, general appearance dull. Head distinctly longer than broad. Occipital margin shallowly concave with rounded corners. Head sides feebly convex in full-face view. Eyes oval, relatively large (EL 0.23-0.24X HW) with eleven ommatidia in the longest row. Median portion of anterior clypeal margin concave. Mandibles longitudinally striate and shining. Antennal scapes, when laid straight back from their insertions, just reach occipital margin. Antennae with dense pubescence. Promesonotum straight in profile. Metanotal groove distinct in profile. The propodeal dorsum feebly depressed medially without sharp lateral margins. Petiole node high and pointed in profile. Postpetiole node rounded and much lower than petiole node. Head dorsum, mesosoma and waist with distinct shagreenate granular sculpture. Dorsal head, mesosoma, waist and gaster lacking standing hairs of any descriptions. Few short hairs in front of eyes, one or two long hairs on head ventrum, gastral ventrum with long hairs. Gaster very finely and densely shagreenate.

Remarks. This new species is a member of the Monomorium salomonis-group as defined by Bolton (1987) and cannot be matched with any of the Arabian Monomorium species in Collingwood (1985), Collingwood \& Agosti (1996), or with the Afrotropical species in Bolton (1987).

Monomorium moathi sp. $\mathrm{n}$., is a species morphologically similar to $M$. areniphilum Santschi, 1911. Both having the following characters: median portion of mesonotum flat to shallowly convex, mesosoma without hairs, propodeal furrow present and distinctly margined, highest point of propodeal dorsum behind metanotal groove on a much lower level than the highest point of the promesonotum.

But a number of consistent differences were found which make it possible to identify and separate them apart. Monomorium moathi is consistently smaller, TL 2.41-2.48 versus TL 3.1-4.30 in areniphilum. Monomorium moathi has a lower head length, HL 0.61-0.65 versus HL 0.86-1.04; has a lower scape length, SL 0.46-0.52 versus SL $0.68-0.88$, has smaller eyes EL $0.23-0.24 \mathrm{X}$ HW versus $0.30-0.35 \mathrm{X}$ HW in areniphilum. Dorsum of head in $M$. moathi without hairs except few short ones in front of eyes, whereas in areniphilum head dorsum with 1-2 pairs of hairs which straddles the midline. In addition, petiole and postpetiole in $M$. moathi are bare, while in $M$. areniphilum petiole has one pair and postpetiole with 2-3 pairs of backwards directed hairs. Moreover, head dorsum in M. moathi is finely shagreenate or shagreenate granulate, whereas in $M$. areniphilum head dorsum with fine dense reticulate to reticulate-shagreenate sculpture. Additionally, metanotal groove in $M$. moathi distinct but not deep, while in $M$. areniphilum metanotal groove conspicuously impressed and deep.

Etymology. This species is named after the friend of Prophet Mohammed, Moath Ebn Jabal who was sent to Yemen to distribute Islam.

\section{ACKNOWLEDGEMENTS}

The authors are most grateful to Dr Brian Taylor and Mr. Barry Bolton for their continuous assistance. Special thanks go to the following: Prof. Hassan Fadl for his comments on the manuscript, Mr. Mohammed F. Sallam for help with species photographing and Mr. Hassan Badri for technical assistance. This paper is part of team effort of Economic Entomology Research Unit (EERU), Plant Protection Department, College of Food and Agriculture Sciences, King Saud University. Mostafa Sharaf is most grateful to his wife Mrs. Amal M. Elsaadany for her encouragement. 


\section{REFERENCES}

Bolton, B. (1987). A review of the Solenopsis genus group and revision of Afrotropical Monomorium (Hymenoptera: Formicidae). Bulletin of the British Museum (Natural History) Entomology. 54: 263-452.

Bolton, B. (1995). A new General Catalogue of the Ants of the World. Harvard University Press, Cambridge, Massachusetts. 504 pp.

Brown, W. L. JR. (2000). Diversity of ants. In: Agosti et al. (Eds), Ants. standard methods for measuring and monitoring biodiversity. Biological diversity hand book series. Smithsonian institution press, Washington and London. 280 pp.

Collingwood, C. A. (1985). Hymenoptera: Fam. Formicidae of Saudi Arabia. Fauna of Saudi Arabia. 7: 230-301.

Collingwood, C.A. and Van Harten, A. (1994). A general guide to the ants Hymenoptera: Formicidae) of Yemen. Yemeni-German plant protection project. $38 \mathrm{pp}$.

Collingwood, C. A. and Agosti, D. (1996). Formicidae (Insecta: Hymenoptera) of Saudi Arabia (part 2). Fauna of Saudi Arabia. 15: 300-385.

Collingwood, C. A. and Van Harten, A. (2001). Additions to the ant fauna of Yemen (Hymenoptera: Formicidae). Esperiana. 8: 559-568.

Collingwood, C. A.; Pohl, H.; Güsten, R.; Wranik, W.; and Van Harten, A. (2004). The ants (Insecta: Hymenoptera: Formicidae) of the Socotra Archipelago. Fauna of Arabia. 20: 473-495. 
Monomorium moathi sp. n., a new ant species from Yemen related to the salomonis-group 41

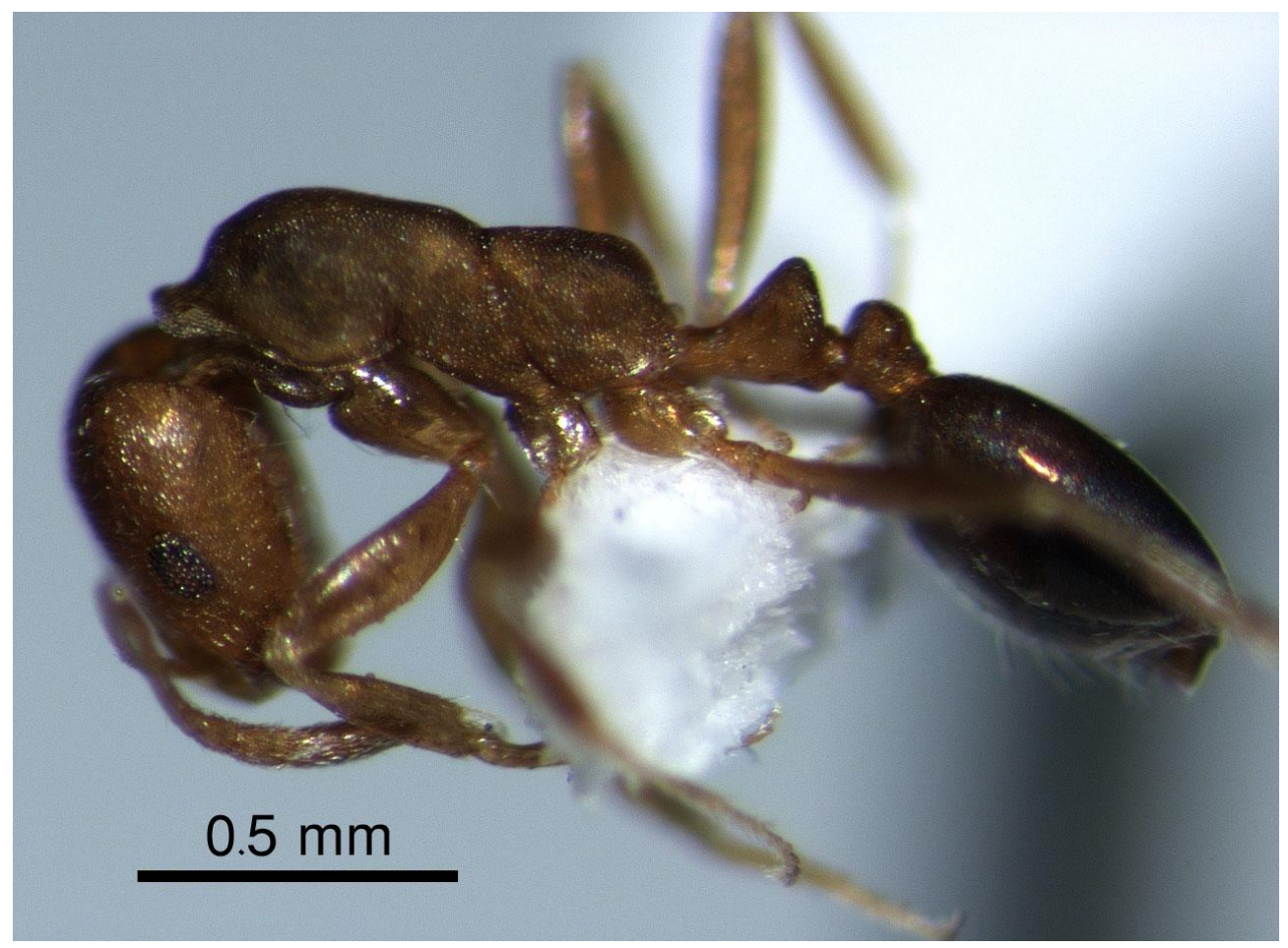

Fig. 1: Profile of Monomorium moathi sp. n. (Holotype, KSMA).

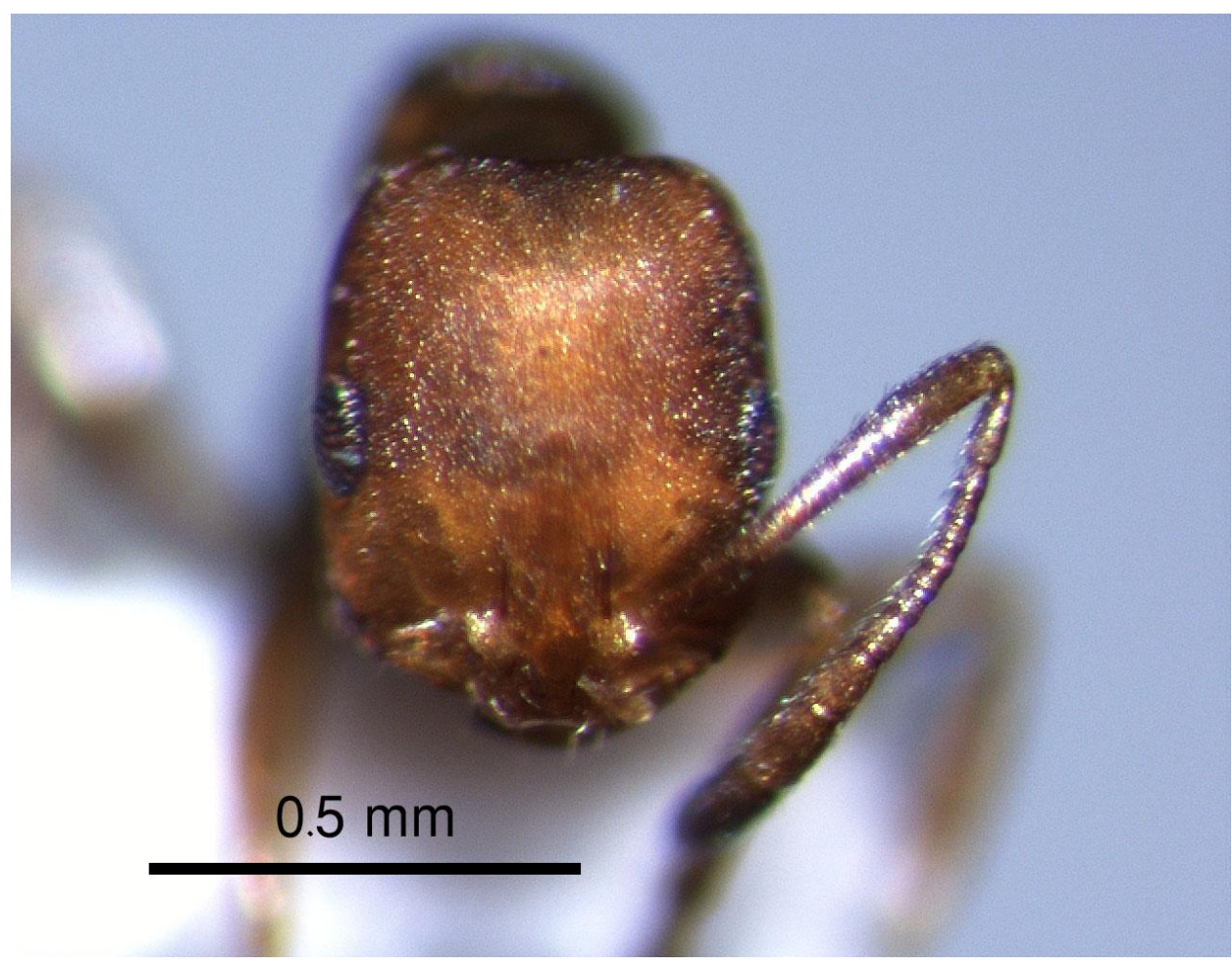

Fig. 2: Full-face view of Monomorium moathi sp. n. (Holotype, KSMA). 


\section{ARABIC SUMMARY}

نوع جديد من النمل Monomorium moathi من اليمن (رتبة غشائية الأجنحة_ فصيلة النمليات) ينتمي إلى

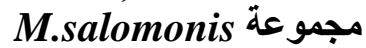

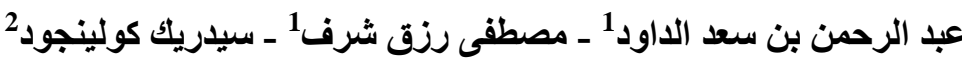

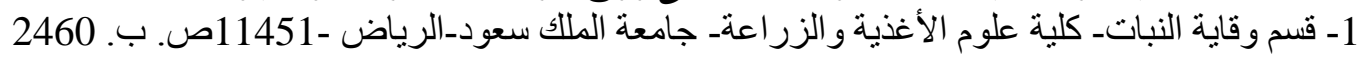

2- 18 Milton street, Skipton, North Yorkshire, BD 23 2ED, U.K.

تم وصف وتوضيح بالرسم النوع الجديد Monomorium moathi من اليمن اعتماداً على أفراد

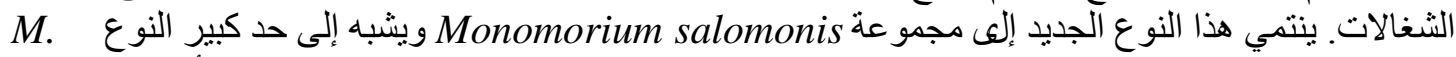
و areniphilum Santschi, 1911

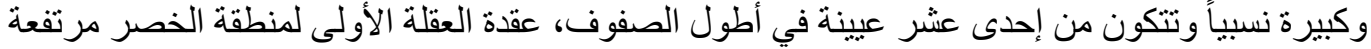

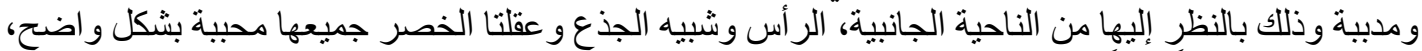

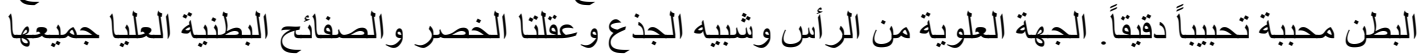

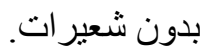

